\title{
Subgrid liquid flux and interface modelling for LES of atomization
} Javier Anez ${ }^{1}$, Aqeel Ahmed ${ }^{1}$, Stefano Puggelli², Julien Réveillon ${ }^{1}$, Jorge Brandle de Motta1, F.X. Demoulin*1

${ }^{1}$ CNRS CORIA UMR 6614, University of Rouen, Technopôle du Madrillet, B.P. 12, 76801 Saint-Etienne-du-Rouvray Cedex, France

${ }^{2}$ Department of Industrial Engineering, University of Florence, Via di Santa Marta 3, 50139 Florence, Italy

${ }^{*}$ Corresponding author: demoulin@coria.fr

\begin{abstract}
Traditional Discrete Particle Methods (DPM) such as the Euler-Lagrange approaches for modelling atomization, even if widely used in technical literature, are not suitable in the near injector region. Indeed, the first step of atomization process is to separate the continuous liquid phase in a set of individual liquid parcels, the so-called primary break-up. Describing two-phase flow by DPM is to define a carrier phase and a discrete phase, hence they cannot be used for primary breakup. On the other hand, full scale simulations (direct simulation of the dynamic DNS, and interface capturing method ICM) are powerful numerical tools to study atomization, however, computational costs limit their application to academic cases for understanding and complementing partial experimental data. In an industrial environment, models that are computationally cheap and still accurate enough are required to meet new challenges of fuel consumption and pollutant reduction. Application of DNS-ICM methods without fairly enough resolution to solve all length scales are currently used for industrial purpose. Nevertheless, effects of unresolved scales are generally cast aside. The Euler-Lagrange Spray Atomization model family (namely, ELSA, also call, $\Sigma-Y$ or $\Omega-Y$ ) developed by Vallet and Borghi pioneering work [1], and [2], at the contrary aims to model those unresolved terms. This approach is actually complementary to DNS-ICM method since the importance of the unresolved term depends directly on mesh resolution. For full interface resolution the unclosed terms are negligible, except in the far-field spray when the unresolved terms become dominant. Depending on the complexity of the flow and the available computational resources, a Large Eddy Simulation (LES) formalism could be employed as modelling approach. This work focus on the two main terms that drive these different modelling approaches namely the subgrid turbulent liquid flux and the resolved interface. Thanks to the open source library OpenFoam ${ }^{\circledR}$ this work is an attempt to review and to release an adapted modelling strategy depending on the available mesh resolution. For validation, these solvers are tested against realistic experimental data to see the overall effect of each model proposal.
\end{abstract}

\section{Keywords}

Euler-Lagrange Spray Atomization model (ELSA), VOF, Sub-grid scales, QME.

\section{Introduction}

From the initial work of Vallet and Borghi [1], different models based on Eulerian modelling for atomization have been studied. Later on, Blokkeel et al. [3] working with the same team completed the original approach by a Lagrangian description of the spray once the primary break-up is achieved. In addition, they proposed to call this approach ELSA for Eulerian-Lagrangian Spray Atomisation model to simplify its denomination, but other names are still in used such as $\Sigma-Y, \Omega-Y$ or $E S A$, depending on which variable has been retained or whether the Lagrangian phase has been activated or not. Moreover, a new model has been attached to this approach such as Quasi-Multiphase Euler flow [4] approach and LES approach [5]. All in all, they belong to the ELSA family of models that try to consider and to model the two main terms that drive atomization process for non-fully resolved cases: the subgrid/unresolved turbulent liquid flux $R_{l i}$ and the unresolved liquid gas interface that will be characterised in this work by $\Sigma$ (area per unit of volume). For brevity the interested reader is referred to [18]. The purpose of this work is to release the model thanks to the open library OpenFoam ${ }^{\circledR}[6]$. Thus a mathematical description is proposed, the solver released will allow to check the numerical methods that must be adapted depending on the modelling proposal. Following the important efforts that have been provided to get data for validation purpose in the ECN workshop framework [7]-[9], the test case designated Spray-A is considered.

\section{Modelling Approaches}

The key point of the proposed ELSA model is the analogy between atomization and turbulent mixing of a jet with large density difference with the ambient medium [1], [2]. This single-phase approach allows the choice of both 
carrier and discrete phases to be avoided [5]. Therefore, the two-phase flow is studied as a single-phase turbulent flow composed of two species with highly variable density. Several features are considered: large-scales properties such as liquid penetration and small-scales characteristics (mean droplet diameter and their size distribution). In this section, starting from this complete approach, governing equations are presented:

$$
\begin{aligned}
& \frac{\partial \rho}{\partial t}+\frac{\partial \rho u_{j}}{\partial x_{j}}=0 \\
& \frac{\partial \rho u_{i}}{\partial t}+\frac{\partial \rho u_{j} u_{i}}{\partial x_{j}}=-\frac{\partial p}{\partial x_{i}}+\frac{\partial}{\partial x_{j}}\left(\rho v\left(\frac{\partial u_{i}}{\partial x_{j}}+\frac{\partial u_{j}}{\partial x_{i}}-\frac{2}{3} \delta_{i j} \frac{\partial u_{k}}{\partial x_{k}}\right)\right)+\rho g_{i}+F_{\sigma}
\end{aligned}
$$

Those equations have to be completed by equation of state and jump relation across the phases [13]. $F_{\sigma}$, is the additional force due to the surface tension depending on the local curvature of the interface. To compute this force and to apply the jump of any variable, the most accurate ICM-DNS code applies direct numerical schemes based on interface reconstruction, along with the numerical mesh characteristics. For instance, the ARCHER code [14] is based on couplet VOF-Level set method for interface reconstruction together with a ghost-fluid approach to represent accurately the discontinuity of variables such as density, pressure and viscosity at the interface. This reconstruction process generally depends on the mesh geometry, hence body-fitted methods based on unstructured mesh are used to address complex geometry. For such complex meshes there is not yet available allpurpose reconstruction methods and lower order approach are applied instead. As for instance, diffusive methods are designed to smear the interface over several mesh cells to recover a continuous behaviour of any variable. Therefore, to reconstruct the field and to localize the interface a phase indicator is used. Among the possible indicator the liquid volume fraction $\left(\alpha_{l}\right)$ has been used mostly due to its conservative form and can be written allowing to ensure the complete conservation of the total amount of liquid during the atomization process. The equation, for constant liquid and gas density reads:

$\frac{\partial \alpha_{l}}{\partial t}+\frac{\partial u_{j} \alpha_{l}}{\partial x_{j}}=0$

Unresolved scales are considered by filtering equation 3 under LES or averaged under RANS framework or simply because the mesh is not fine enough to capture all the fluctuations (i.e., When values integrated over a cell are smeared while discretizing with finite volume method). The accuracy of different RANS turbulence models when applied to two-phase flow was previously studied [23]. All these operators when applied to the previous equation will produce additional terms. First let's consider the LES filtered operation for liquid volume fraction, then equation 3 becomes

$\frac{\partial \overline{\alpha_{l}}}{\partial t}+\frac{\partial \overline{u_{j}} \overline{\alpha_{l}}}{\partial x_{j}}=\frac{\partial R_{l j}}{\partial x_{j}}=\frac{\partial \overline{u_{j} \alpha_{l}}-\overline{u_{j}} \overline{\alpha_{l}}}{\partial x_{j}}=\frac{\left.\partial \overline{u_{\jmath}}\right|_{l} \overline{\alpha_{l}}-\left(\left.\overline{\alpha_{l}} \overline{u_{\jmath}}\right|_{l}+\left.\overline{\alpha_{g}} \overline{u_{\jmath}}\right|_{g}\right) \overline{\alpha_{l}}}{\partial x_{j}}=\frac{\partial \overline{\alpha_{l}}\left(1-\overline{\alpha_{l}}\right)\left(\left.\overline{u_{j}}\right|_{l}-\left.\overline{u_{\jmath}}\right|_{g}\right)}{\partial x_{j}}$

Where the liquid flux, $R_{l j}$ appears (first term on RHS) and is related to the unresolved part of the velocity that is known to produce additional diffusion. This part can be modeled by a turbulent diffusion process [22]. Another feature of this term appears on the last term, where the slip velocity $\left(\left.\bar{u}_{j}\right|_{l}-\left.\bar{u}_{J}\right|_{g}\right)$ is clearly related to $R_{l j}$. This characteristic of multi-phase flow is well known, see for example the work of Simonin et al. [16]. He defined the drift and slip velocity between two phases. For atomization processes this effect might be of some importance, thus two modelling approaches should be developed, namely the basic ELSA approach (only drift is considered) and the Quasi Multiphase Eulerian method, QME [4]. Therefore, the turbulent diffusion liquid flux rewrites:

$\frac{\partial R_{l j}}{\partial x_{j}}=\frac{\partial \overline{\alpha_{l}}\left(1-\overline{\alpha_{l}}\right)\left(\left.\overline{u_{j}}\right|_{l}-\left.\overline{u_{j}}\right|_{g}\right)}{\partial x_{j}}=\frac{\partial \overline{\alpha_{l}}\left(1-\overline{\alpha_{l}}\right) \bar{V}_{r l g j}}{\partial x_{j}}=\frac{\partial \overline{\alpha_{l}}\left(1-\overline{\alpha_{l}}\right)\left(\bar{u}_{\text {slip } j}-\bar{v}_{d r i f t}\right)}{\partial x_{j}}$

\section{Remarks on averaging}

Considering the simple case of constant gas and liquid density, $\rho_{g}$ and $\rho_{l}$, respectively, the filtered density, $\bar{\rho}=$ $\rho_{l} \bar{\alpha}_{l}+\left(1-\bar{\alpha}_{l}\right) \rho_{g}$, is far for being constant and some correlations should be addressed. To reduce closure issues Favre averaging/filtering is employed, which defines for any variable $\tilde{\varphi}=\frac{\overline{\rho \varphi}}{\bar{\rho}}$. This approximation is used widely for single-phase flow with variable density, hence it can be applied for two-phase flow following the modeling approach explained above. Indeed in their original work, Vallet and Borghi [1] have used the Favre averaging. Additionally, the resulting Favre velocity fluctuation is usually related to the turbulent liquid mass flux [22]. However, two 
difficulties arise from this approach. Firstly, any Favre-averaged variable is pondered by the density. Figure 1 shows the Favre-averaged velocity mixture with respect to the liquid volume fraction $\overline{\alpha_{l}}$, for constant liquid and gas velocity, $u_{l}$, and $u_{g}$, respectively. The nonlinear relation of the Favre averaging, namely $\tilde{u}$, is enhanced as the density ratio grows, $r$. For instance, when $r=100$, and the mixture is composed by $90 \%$ of gas $\left(\bar{\alpha}_{l}=0.1\right)$, the Favre-averaged velocity mixture is still mainly related to the liquid velocity. On the other hand, Reynolds-averaged mixture velocity exhibits linear behavior between the limiting cases (pure liquid or pure gas). Secondly, the Reynolds-averaged velocity field is by definition divergence free, on the contrary for the Favre-averaged velocity field, as shown below:

$\rho_{l}=c s t, \rho_{g}=c s t \rightarrow \frac{\partial u_{j}}{\partial x_{j}}=0 \rightarrow \frac{\partial \overline{u_{j}}}{\partial x_{j}}=0 \rightarrow \frac{\partial \widetilde{u_{j}}}{\partial x_{j}}=-\frac{1}{\bar{\rho}} \frac{D \bar{\rho}}{D t}$

These two Favre averaging drawbacks compel us to consider directly Reynolds averaging formulation together with liquid volume fraction (volume formulation) field, instead of liquid mass fraction (mass formulation). The motivation is also to get closer to VOF approaches. Our goals are twofold, firstly to compare how volume formulation matches with well-established results based on mass formulation [22], and secondly to develop an ELSA model compatible with ICM filtered by LES turbulence model.

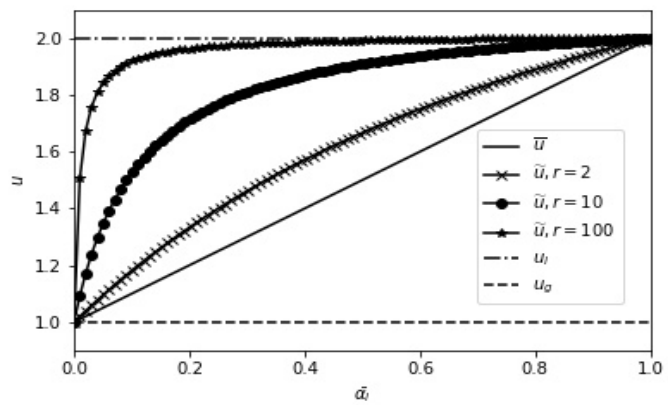

Figure 1: Plot of Favre-averaged velocity and Reynolds-averaged velocity for different density ratio $r=\frac{\rho_{l}}{\rho_{g}}$ as a function of the liquid volume fraction $\left(u_{l}=2, u_{g}=1\right)$.

LES formulation compatible with ICM.

For full scale resolution, ICM method aims at keeping a sharp interface, thus a discontinuous profile of $\alpha_{l}$ across the phases exits in particular during the convection process. This property is either directly included in the numerical scheme (VOF, Level-Set, ghost-fluid, among others) or obtained by additional correction designed to prevent numerical diffusion that could smear the $\alpha_{l}$ profile. The interFoam solver of OpenFOAM® is based on this last technique, where Weller [6] proposed to use an additional flux of liquid directed toward the interface proportional to the local velocity magnitude $\left(u_{r}\right)$ and located only where a mixture of liquid and gas exist (i.e. $\alpha_{l} \in[0,1]$ ). This method is often referred as the VOF method, even if there is no real reconstruction of the interface. See equation 6 , last term on the left. This equation when is solved using Direct Numerical Simulations (DNS) all fluctuations scales are solved up to the grid level and no averaged filtering is required. On the other hand, following the modelling approach in this study, LES filtering or averaged under RANS framework is used, which in either case, the turbulent diffusion liquid flux is set to zero:

$\frac{\partial \overline{\alpha_{l}}}{\partial t}+\frac{\partial \overline{u_{j}} \overline{\alpha_{l}}}{\partial x_{j}}+\frac{\partial u_{r} \overline{\alpha_{l}}\left(1-\overline{\alpha_{l}}\right)}{\partial x_{j}}=0$

Notice that several proposals exist in OpenFOAM® to improve this point in particular the isoAdvector approach [15]. There are many successful examples in the literature of these fully resolved approaches combining ICM method with DNS using mesh resolution high enough to compute all the flow scales. It is important to emphasize that the drift behavior of the unresolved liquid flux $R_{l j}$ is not compatible to the ICM method since the latter assumes the $\alpha_{l}$ profile to be discontinuous. Considering the shortcomings of diffusive interface approaches in the dense spray region and in order to develop a model suitable also in the dilute spray region, a coupling technique between ELSA and the ICM method is proposed, where the turbulent diffusion liquid flux is no longer set to zero. Hence, starting from equations 4,5 and 6 , the liquid volume fraction equation has been modified considering, $C_{\alpha}$ as a pondering coefficient between ELSA-base and an ICM approach. For a complete definition of $C_{\alpha}$, the interested reader is addressed to [17]. Clearly, $R_{l j}$ can be modeled using drift and slip velocity between the phases as explained above. The final assembled model for all scales is presented below: 
$\frac{\partial \overline{\alpha_{l}}}{\partial t}+\frac{\partial \overline{u_{j}} \overline{\alpha_{l}}}{\partial x_{j}}+\frac{\partial C_{\alpha} u_{r} \overline{\alpha_{l}}\left(1-\overline{\alpha_{l}}\right)}{\partial x_{j}}=\left(1-C_{\alpha}\right) \frac{\partial \overline{\bar{\alpha}_{l}}\left(1-\overline{\alpha_{l}}\right)\left(\bar{u}_{\text {slip } j}-\bar{v}_{d r i f t}\right)}{\partial x_{j}}$

The advantages of the proposed model is to determine a resolution of the interface with ICM in a limited region, whereas $R_{l j}$ prevails when the fluctuations between phases become significant at subgrid-scale.

\section{Numerical model}

Regarding the geometry and the inflow condition several options may be considered, full 3D simulation with in injector flow and needle movement [10] up to a simple 2D axisymmetric configuration [11]. On both cases it has been possible to show that even with the less resolved configuration (RANS) the essential features of the injection can be captured with the ELSA family. An advantage of this test case is the possibility to simulate also the flow inside the injector. A simple geometry consisting of only spray domain is also studied in addition to full injector flow modelling, to visualize more clearly different aspects of spray models. An example of the mesh used for this simulation are presented on Figure 2. The 1D axial profile of injector (210675) is taken from ECN website [9] (red line on the figure). Using GMSH [12] the geometry was extended to include injector sac and needle. Then a structured mesh, consisting of 17168 hexahedral cell types was obtained with 10 cells at the exit of the injector along the radial direction. Finally an axisymmetric case was setup. Another simple spray chamber geometry is also tested (see Figure 1, bottom). For 3D simulation either the geometry is rotational extruded from the axisymmetric case, or the full 3D surface available from ECN website is used. In this latter case because of uncertainties on the real geometry of the injector, and despite several measurements, the shape profile of the $2 \mathrm{D}$ axisymmetric injector pipe is preserved up to 10 diameters of the orifice.

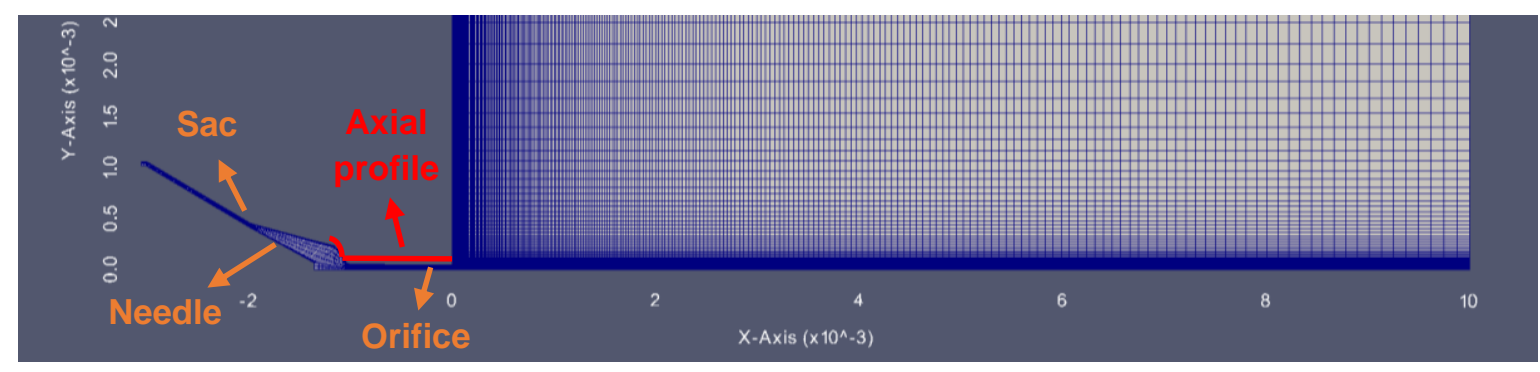

Figure 2. Structured mesh with in injector pipe.

In reality, the injector includes also an axial displacement of the needle, however, despite its potential effect on the flow behaviour it has been chosen not to be considered in this study. By using a transient mass flow rate allows to reproduce partly the effect of the needle motion. Nonetheless, the established injection jet can be obtained directly with a constant mass flow rate. The first comparison will be based on this simplified test case. Accordingly, operating conditions are reported in Table 1.

Table 1. Conditions for non-evaporating ECN Spray-A [9]

\begin{tabular}{ll}
\hline Fuel & $\mathrm{n}$-Dodecane \\
Ambient composition & $100 \% \mathrm{~N} 2$ \\
Injection pressure $[\mathrm{MPa}]$ & 150 \\
Ambient temperature $[\mathrm{K}]$ & 303 \\
Ambient density $\left[\mathrm{kg} / \mathrm{m}^{3}\right]$ & 22.8 \\
Fuel injection temperature $[\mathrm{K}]$ & 343 \\
\hline
\end{tabular}

\section{Results and discussion}

A series of modifications is proposed, based on the physics to be modelled. Whether the interface is resolved or sub-grid scales dominate or relative velocity between the phases occurs, such as the one encountered in flow separation. Firstly elsaBase is presented, which solves the turbulent diffusion liquid flux term $\left(R_{l j}\right)$ including only the drift component, and with an additional scalar transport equation for the liquid-gas surface interface $(\Sigma)$. Secondly, elsaBaseQme, which solves the same equations as ElsaBase with the added slip component of the turbulent diffusion liquid flux $\left(\bar{u}_{\text {slip }}-\bar{u}_{\text {drift }}\right)$. Thirdly, interRansElsa, which is an adapted version of the equation 7 , mainly to be used for 2D axisymmetric geometries, and without the slip part of the diffusion term. Finally, 
interLESElsaBase, which solves equation 7 for 3D complex geometries. Different elsa models are summarized in Table 2.

Table 2. Different ELSA models

\begin{tabular}{lcccc} 
Solver & $\begin{array}{c}\text { Turbulent } \\
\text { diffusion flux }\end{array}$ & $\begin{array}{c}\text { Slip } \\
\text { velocity }\end{array}$ & $\begin{array}{c}\text { Interface } \\
\text { compression term }\end{array}$ & $\begin{array}{c}\text { LES } \\
\text { filtered }\end{array}$ \\
\hline interFoam & & & $\checkmark$ & \\
elsaBase & $\checkmark$ & & & \\
elsaBaseQme & $\checkmark$ & $\checkmark$ & & \\
interRansElsa & $\checkmark$ & & $\checkmark$ & $\checkmark$ \\
elsaBaseLes & $\checkmark$ & & &
\end{tabular}

\section{Validation}

A comparison process has been made against experimental and numerical data available from the Engine Combustion Network (ECN) in order to validate the proposed ELSA model. The "Spray-A" non evaporating configuration has been selected, with exact aforementioned fluid properties. The experimental data used for validation include the Projected Mass Density of the fuel (PMD), which was obtained by x-ray radiography measurement techniques [19, 20], and the Transverse Integrated Mass (TIM), which was acquired from the integral of the projected density across a transverse position at a particular axial location [18]. For the numerical data in the present study a fixed needle motion was modeled using a rate of injection generator for Spray-A, http://www.cmt.upv.es/ECN09.aspx. Figure 3 shows the comparison in terms of the PMD and TIM between experimental and numerical data for the first 5 cases described in table 2. Figure 3 on the left displays both limiting cases such as interFoam (interface resolved) and elsaBase (interface diffused), overpredicting and underpredicting liquid penetration, respectively. On the other hand, both elsaBaseLes (with LES filtered) and elsaBase with the round jet correction are able to fairly match the experimental results. Moreover, small differences can be found when the nozzle is added. However, it is believed these differences come from higher mesh resolution used in the nozzle region. Looking closer to the PMD (Figure 3 on the right), based on the radial profile, all models accurately predict the liquid core at $0.1 \mathrm{~mm}$ from the liquid inlet. At this axial position the turbulent instabilities have not yet been developed while using only the chamber without the nozzle, therefore all models should colapse and match interFoam and the case with the nozzle, as shown. On the latter case, the behavior is expected based on the already establishment of the flow inside the nozzle. The differences observed between 0.05 and $0.06 \mathrm{~mm}$ in the radial PMD for all other cases are mainly due to poor mesh resolution which introduces additional numerical errors. On the other hand, at $2 \mathrm{~mm}$ from the inlet, even though RANS models generally tend to overpredict the fuel dispersion, interface instabilities downstream of the flow are comparable with experiments by showing less radial liquid dispersion and higher liquid penetration than previous aforementioned cases when the round-jet correction is used, and even equivalent results are matchable compared with LES case. It is true that LES does not require any turbulent modelling adjustment as the one made with RANS, nevertheless, both models deal the turbulence as incompressible, which is not completely accurate. Another parameter to measure radial dispersion is the TIM which is shown on the right of the figure. ElsaBase clearly represents the model with the highest flow dispersion. While elsaBase filtered by LES, shows the least diffusivity of all, especially downstream of the flow (near $3 \mathrm{~mm}$ ) where the $3 \mathrm{D}$ turbulent instabilities begin to play a role. It is the turbulent modelling the driven mechanism which dominates the diffusion in this area. Besides the mesh resolution, a proper turbulent modelling is imperative to exactly match the experimental results, therefore a compressible approach is a reasonable choice. For instance, the turbulent kinetic energy should be accounted differently between the phases. Being the liquid heavier than the gas, the turbulent should be diffused more rapidly on the gas phase. Such modelling approach is outside the scope of this paper, since these models are based on the mixture properties. The purpose of the paper is to present different approaches based on ELSA developed so far, the majority of which are able, with minimal computational efforts by using RANS, to offer comparable experimental results within the near nozzle region.

In figure 4 results are shown using 2D axisymmetric geometry with the nozzle for all ELSA versions. Liquid volume fraction and velocity fields are displayed. At the top row of images, interFoam displays a continuous liquid penetration all the way to the exit of the chamber, showing no diffusion as anticipated, which clearly does not correspond with experiments. Hence the need of an alternative, diffusive model to accurately model the liquid dispersion demonstrated with $\mathrm{x}$-ray radiography. 
$\mathrm{PMD} \mu \mathrm{g} / \mathrm{mm}^{2}$
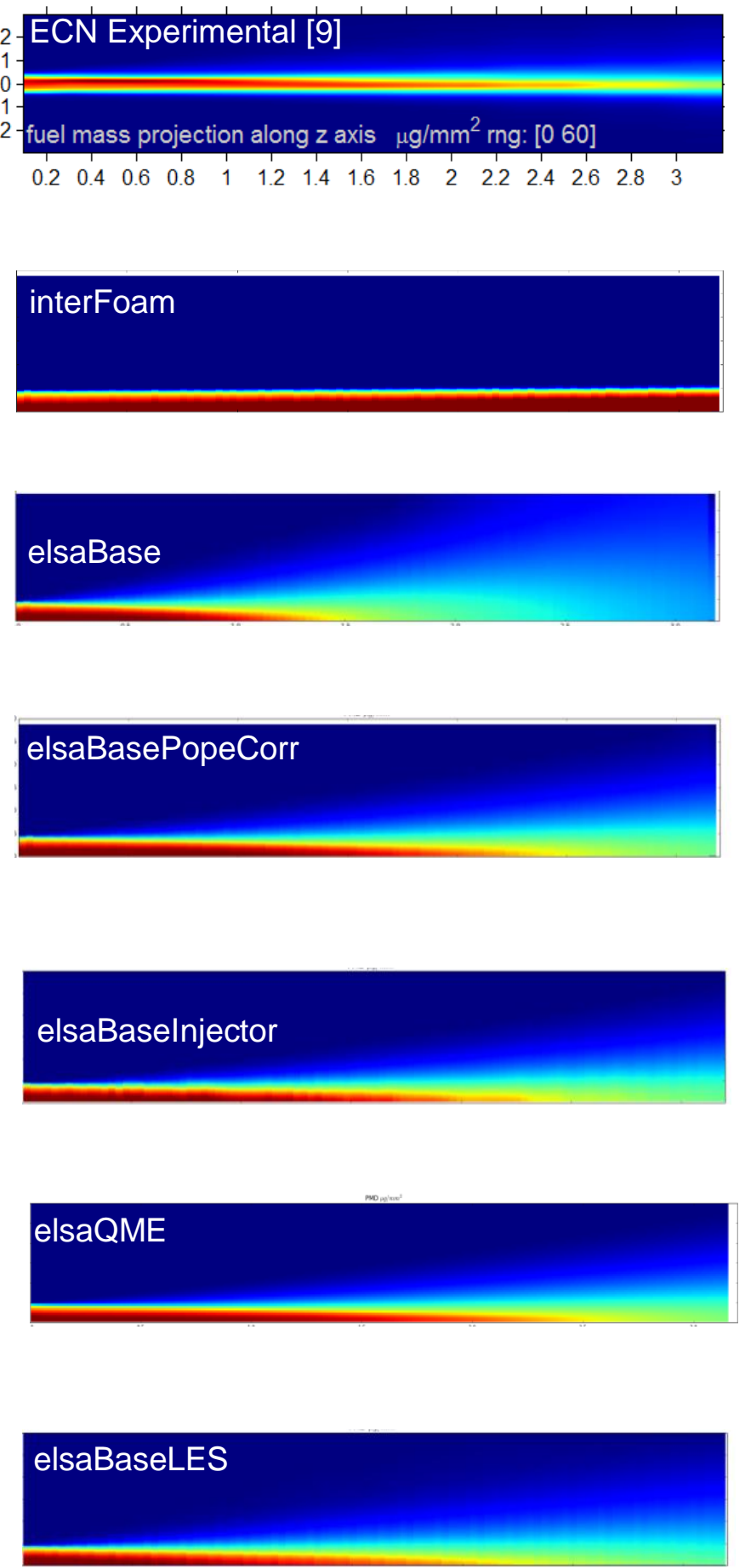
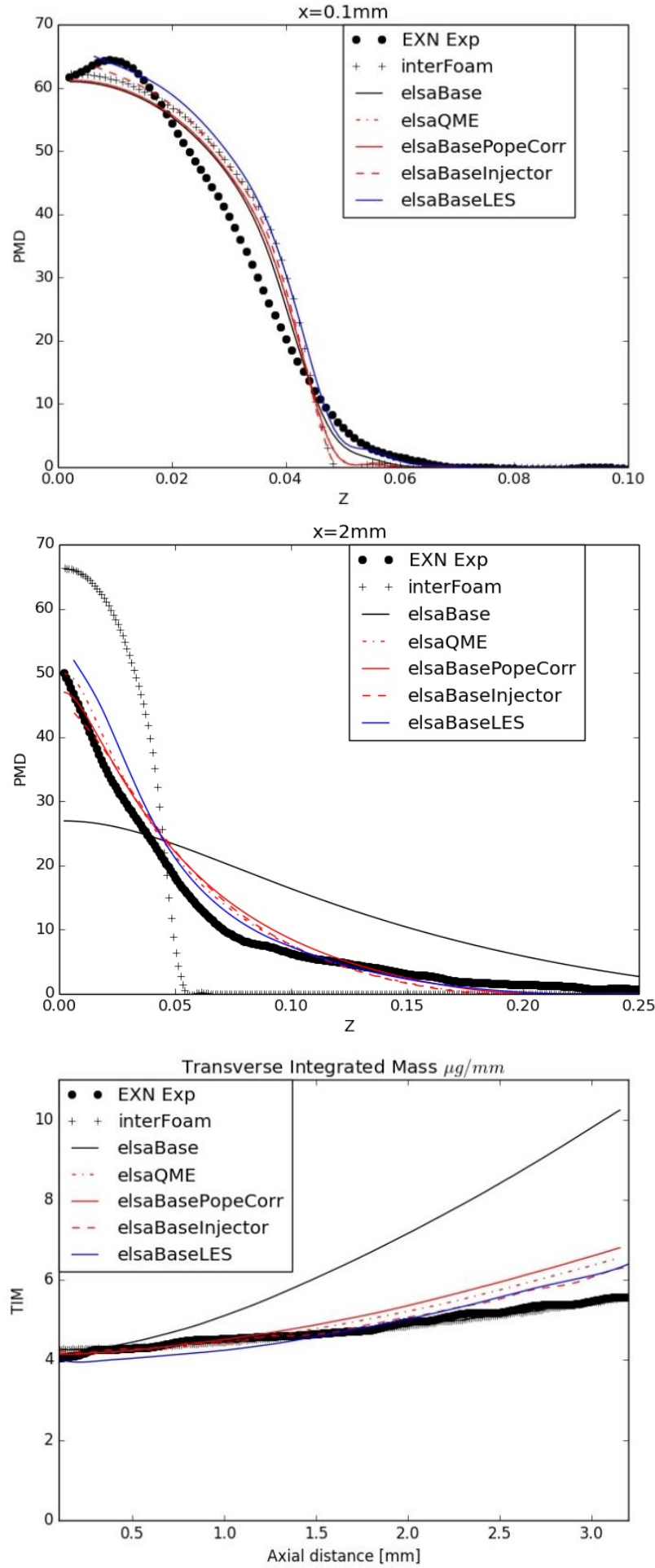

Figure 3. Experimental and numerical Projected Mass Density fields at $0.1 \mathrm{~ms}$ after start of injection (elsaBaselnjector was at $0.5 \mathrm{~ms}$ ) (left). PMD radial profiles at axial location $0.1 \mathrm{~mm}, 2 \mathrm{~mm}$ and TIM (right).

Then, elsaBase and elsaBaseQme model, second and third row of images, respectively, show as expected high diffusivity of the interface, but falls short reproducing the liquid core penetration, with just a little difference between both models on the radial liquid dispersion hardly detectable at the local map span, and especially at the velocity fields. ElsaBase ( $\mathrm{k}-\epsilon$ modified) shows an increase of liquid penetration due to lower diffusivity tuned by the corrected parameter, however, the diffusion is still overpredicted by the lack of appropriate ICM. What is more, an attempt to 
unify both interface resolution physics has been achieved by using, interRansElsa. However, results illustrate no significance difference between ElsaBase, elsaBaseQme, and interRansElsa, mainly due to inappropriate mesh resolution. It is believed, improvements of mesh quality would arise to better liquid penetration, and dispersion to match experimental results by properly capture the interface at the vicinity of nozzle exit, especially before instabilities on the liquid surface due to the turbulent interactions with the gas generates the breakup process. Finally, a refinement meshing process is being held along with 3D Spray-A model reconstruction, both are estimated to be shown and compared at the conference.
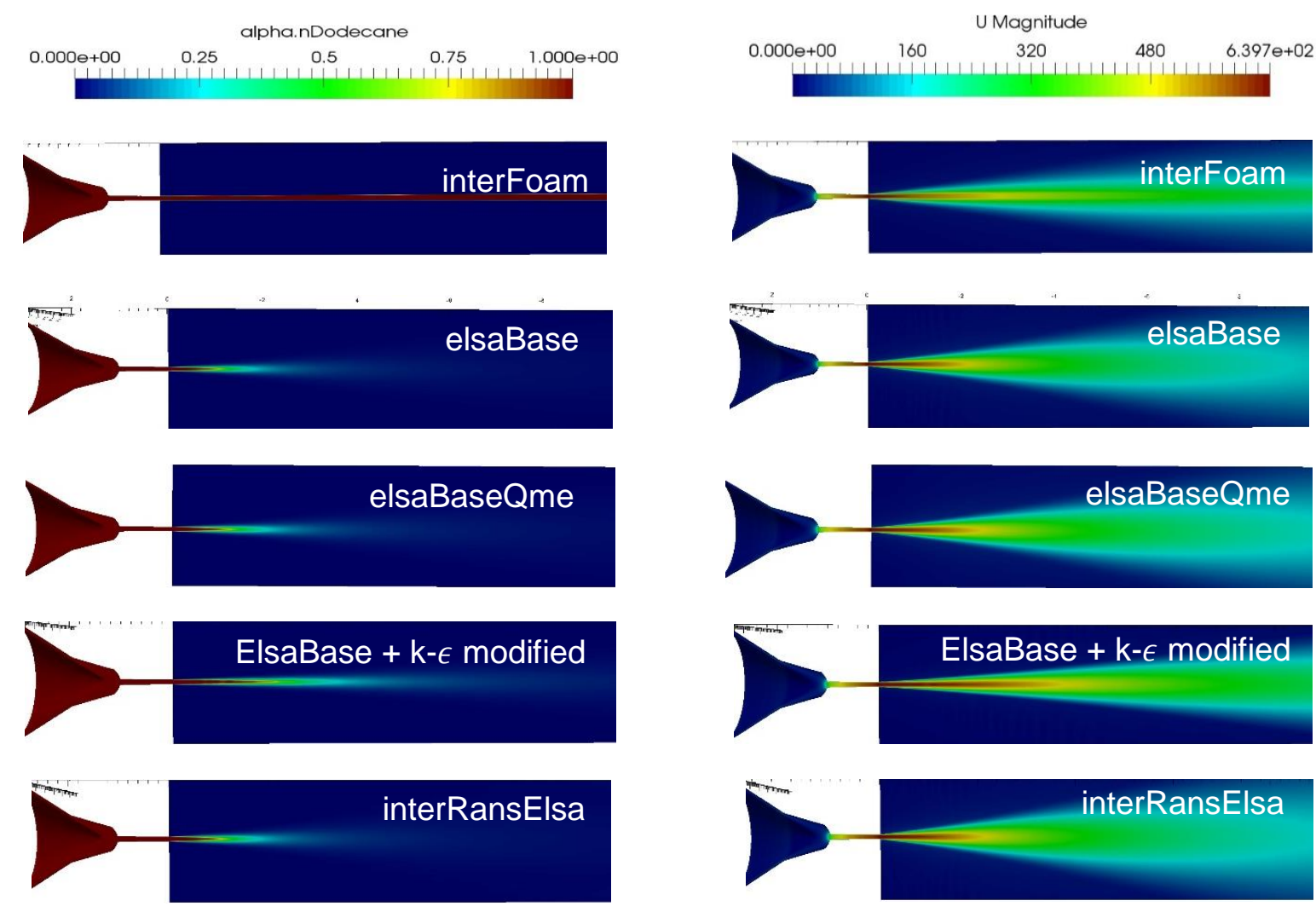

Figure 4. Liquid volume fraction and velocity fields for different ELSA family models, and interFoam, namely, ElsaBase, interRansElsa, elsaBaseQME, and eslaBase with additional modification.

Using second order closure, a slip velocity between the high velocity liquid jet and the quiescent ambient is generated with elsaBaseQME. The relative velocity between the two phases is shown in Figure 5 . It takes its maximum in the near injection region, where the liquid core is still not affected by the breakup process: locally, the liquid presents a liquid volume fraction between 0.6-0.8 and tends to accelerate the gas phase and, due to the air inertia, to generate a relative flux. Even if such values of slip velocity are small with respect to the liquid velocity, a reduction of turbulent diffusion in radial direction with respect to elsaBase can be clearly appreciated. It is interesting to point out that including these effects, the results obtained with elsaBaseQME and interRansElsa are really similar. Going further downstream, the slip velocity is progressively reduced by the drag effects, which, based on the local dynamic relaxation time, lead to similar values of gas and liquid velocity. Clearly, such evolution of the relative flux is directly related to the atomization process, which is shown in Figure 6 for the three ELSA solvers.

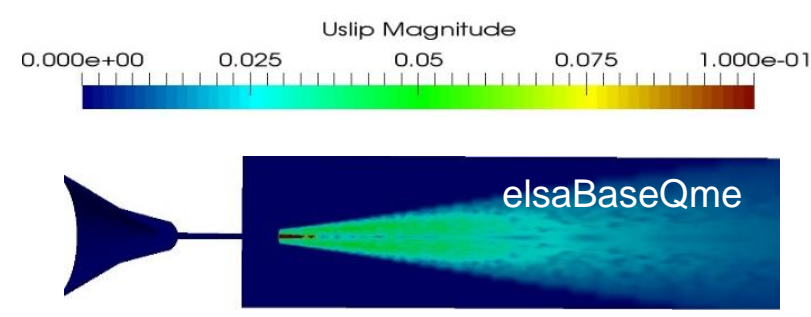

Figure 5. Relative velocity field from elsaBaseQme. 
It is worth pointing out that for interFoam has no information about the evolution of the interface, which is no more available as long as the interface is not sharply resolved. All the proposed versions of ELSA leads to a similar characterization of the atomization process. The liquid jet, due to its high Weber and Reynolds numbers, undergoes a quick atomization process, which is pointed out by the zone where the production of $\Sigma$ is really high. Such violent atomization is related to the growth of instabilities on the liquid surface due to the turbulent interactions with the gas phase in the near injection region. A spray is therefore generated and immediately relaxed to the gas velocity.

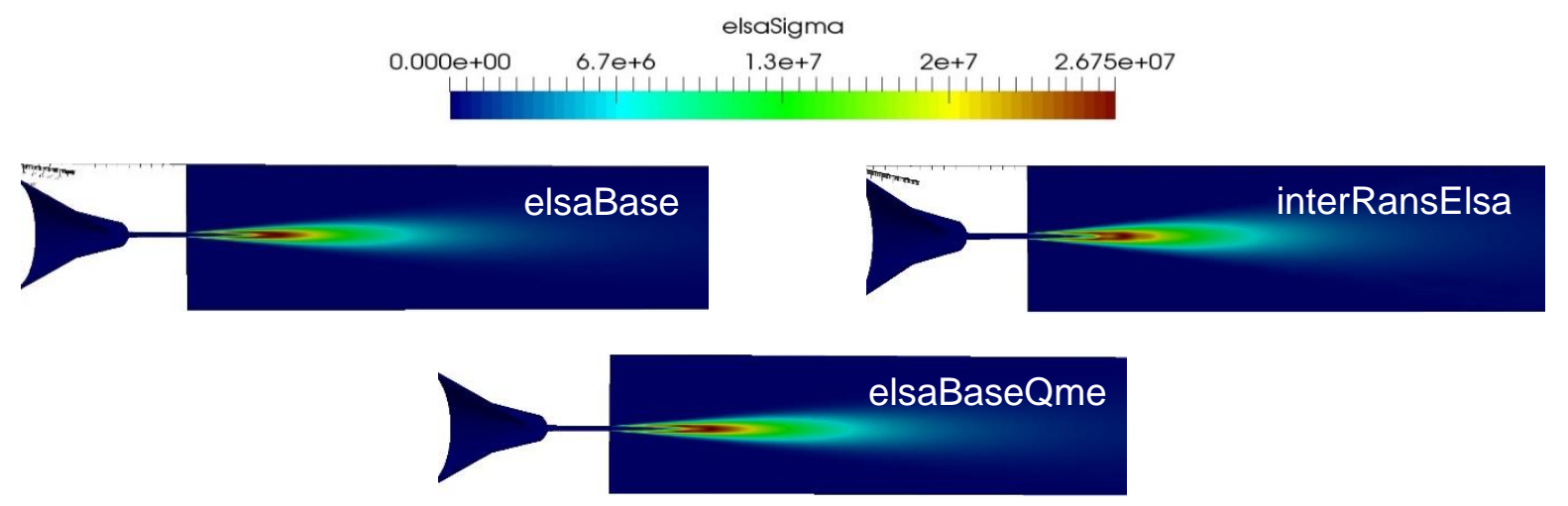

Figure 6. Liquid Gas interface density fields for different ELSA family models, namely, ElsaBase, interRansElsa, and elsaBaseQME.

\section{Conclusions}

The main aim of the present study was to propose a review of the capabilities of several modelling strategies for atomization processes belonging to the ELSA framework. Here, the two-phase flow is studied as a single-phase turbulent flow composed of two species with highly variable density. Starting from the basic approach (i.e. elsaBase), two different extensions based on including the slip velocity effects (i.e. elsaBaseQME) and in dealing with the dense spray region through a ICM (i.e. interRansElsa) are presented. All these approaches have been tested on an experimental test case representative of diesel injection (namely, Spray-A non-evaporating conditions) directly taken from the Engine Combustion Network (ECN). Results obtained on a 2D geometry have been here reported, even if the real $3 \mathrm{D}$ geometry directly taken from the $\mathrm{ECN}$ website is now under investigation. A preliminary comparison both with experimental data and with available numerical results is firstly shown in order to prove that an overall agreement has been achieved. Considering the modelling hypothesis here used, the LES-ELSA approach seems capable of properly reproducing the main features of the analysed test case. Then, a comparison of the performances of the different version of ELSA is reported in order to show the impact of the inclusion of a slip velocity between phases and of an ICM for the dense spray region. Finally, a low-cost atomization modelling approach has been developed suitable from in-flow nozzle injector to far-field spray. What is more, adaptable based on interface resolution, thus avoiding the need to define a carrier phase and a discrete phase. Another important result is the use of RANS modelling instead of LES, can give the same amount of numerical accuracy.

\section{Acknowledgements}

This work was partly supported European Union's Horizon 2020 research and innovation program under the Sklodowska-Curie grant agreement No. 675676. Simulations were carried out at TGCC (The Curie supercomputer, owned by GENCl and operated into the TGCC by CEA), and at CRIHAN (Centre de Ressources de Haute Normandie).

\section{References}

[1] A. Vallet and R. Borghi, "Modélisation Eulerienne de L'atomisation d'un Jet Liquide," C R Acad Sci Paris Sér II B, vol. 327, pp. 1015-1020, 1999.

[2] A. Vallet, A. A. Burluka, and R. Borghi, "Development of a Eulerian model for the 'Atomization' of a liquid jet," At. Sprays, vol. 11, no. 6, pp. 619-642, 2001.

[3] G. Blokkeel, R. Borghi, and B. Barbeau, "A 3d Eulerian model to improve the primary breakup of atomizing jet," SAE Tech. Pap., vol. 2003-01-0005, 2003.

[4] A. Andreini, C. Bianchini, S. Puggelli, and F. X. Demoulin, "Development of a turbulent liquid flux model for Eulerian-Eulerian multiphase flow simulations," Int. J. Multiph. Flow. 
[5] J. Chesnel, J. Reveillon, T. Menard, and F. X. Demoulin, "Large eddy simulation of liquid jet atomization," At. Sprays, vol. 21, no. 9, pp. 711-736, 2011.

[6] H. G. Weller, H. Jasak, and C. Fureby, "A tensorial approach to computational continuum mechanics using object-oriented techniques," Comput. Phys., vol. 12, no. 6, 1998.

[7] A. L. Kastengren et al., "ENGINE COMBUSTION NETWORK (ECN): MEASUREMENTS OF NOZZLE GEOMETRY AND HYDRAULIC BEHAVIOR,” vol. 22, no. 12, pp. 1011-1052, Apr. 2013.

[8] M. Meijer et al., "ENGINE COMBUSTION NETWORK (ECN): CHARACTERIZATION AND COMPARISON OF BOUNDARY CONDITIONS FOR DIFFERENT COMBUSTION VESSELS," vol. 22, no. 9, pp. 777-806, Jan. 2013.

[9] Engine Combustion Network.

[10] Q. Xue et al., "An Eulerian CFD model and X-ray radiography for coupled nozzle flow and spray in internal combustion engines," Int. J. Multiph. Flow, vol. 70, no. 0, pp. 77-88, Apr. 2015.

[11] J. M. Desantes, J. M. García-Oliver, J. Pastor, A. Pandal, E. Baldwin, and D. Schmidt, "Coupled/decoupled spray simulation comparison of the ECN spray a condition with the Sigma- Y eulerian atomization model," Int. J. Multiph. Flow, vol. 80, pp. 89-99, 2016.

[12] C. Geuzaine and J.-F. Remacle, "Gmsh: A 3-D finite element mesh generator with built-in pre-and postprocessing facilities," Int. J. Numer. Methods Eng., vol. 79, no. 11, pp. 1309-1331, 2009.

[13] M. Ishii, Thermo-fluid dynamic theory of two-phase flow. Paris: Eyrolles, 1975.

[14] T. Menard, S. Tanguy, and A. Berlemont, "Coupling level set/VOF/ghost fluid methods: Validation and application to 3D simulation of the primary break-up of a liquid jet," Int. J. Multiph. Flow, vol. 33, no. 5, pp. 510524, 2007.

[15] J. Roenby, H. Bredmose, and H. Jasak, "A computational method for sharp interface advection," R. Soc. Open Sci., vol. 3, no. 11, Nov. 2016.

[16] O. Simonin, "Statistical and continuum modelling of turbulent reactive particulate flows," Lect. Ser. 1996-02 Von Karman Inst. Fluid Dyn., 2000.

[17] Hecht N (2014) Simulation aux grandes 'echelles des 'ecoulements liquide-gaz: application 'a l'atomisation. PhD thesis, University of Rouen.

[18] Kastengren, Alan L., et al. "X-ray radiography measurements of diesel spray structure at engine-like ambient density." Atomization and Sprays 19.11 (2009)..

[19] Kastengren, A. L., et al. "Time-resolved X-ray radiography of diesel injectors from the engine combustion network." ICLASS Paper 1369 (2012).

[20] Pickett, Lyle M., et al. "Comparison of near-field structure and growth of a diesel spray using light-based optical microscopy and x-ray radiography." SAE International Journal of Engines 7.2014-01-1412 (2014): 1044-1053.

[21] Pope, S. B. "An explanation of the turbulent round-jet/plane-jet anomaly."AIAA journal 16.3 (1978): 279-281.

[22] Lebas, Romain, et al. "Numerical simulation of primary break-up and atomization: DNS and modelling study." International Journal of Multiphase Flow 35.3 (2009): 247-260.

[23] Demoulin, Francois-Xavier, et al. "A new model for turbulent flows with large density fluctuations: Application to liquid atomization." Atomization and Sprays 17.4 (2007). 